\title{
Detection and Profiling of Antibiotic Resistance among Culturable Bacterial Isolates in Vended Food and Soil Samples
}

\author{
Susan W. Muriuki, ${ }^{1}$ Johnstone O. Neondo, ${ }^{2}$ and Nancy L. M. Budambula $\mathbb{D}^{1}$ \\ ${ }^{1}$ Department of Biological Sciences, University of Embu, P.O. Box 6-60100, Embu, Kenya \\ ${ }^{2}$ Institute for Biotechnology Research, Jomo Kenyatta University of Agriculture and Technology, P.O. Box 62000-00200, \\ Nairobi, Kenya \\ Correspondence should be addressed to Nancy L. M. Budambula; budambula.nancy@embuni.ac.ke
}

Received 9 March 2020; Revised 13 July 2020; Accepted 20 August 2020; Published 7 September 2020

Academic Editor: Clemencia Chaves Lopez

Copyright ( $) 2020$ Susan W. Muriuki et al. This is an open access article distributed under the Creative Commons Attribution License, which permits unrestricted use, distribution, and reproduction in any medium, provided the original work is properly cited.

\begin{abstract}
The emergence and persistence of antibiotic resistance remain formidable health challenges. This study aimed at detecting and profiling antibiotic resistance of bacterial contaminants in vended food and the environment. Seventy antibiotic-resistant bacterial isolates were isolated from fried fish, African sausages, roasted meat, smokies, samosa, chips (potato fries), vegetable salads, and soil samples collected from Embu Town and Kangaru Market in Embu County, Kenya. The antibiotic susceptibility test, morphological and biochemical characterization, antibiosis assay, polymerase chain reaction-based detection of antibiotic resistance genes, and sequencing of the 16S rRNA gene were done. Analysis of variance on all measured data was done, and Tukey's honest test was used to compare and separate mean diameters of zones inhibition. Resistance of bacterial isolates to antibiotics was chloramphenicol (90\%), cefotaxime (84.29\%), nalidixic acid (81.43\%), tetracycline $(77.14 \%)$, amoxicillin $(72.86 \%)$, gentamycin (48.57\%), streptomycin (32.86\%), and trimethoprim + sulphamethoxazole (30\%). Isolate KMP337, Salmonella spp., exhibited highly significant antibiosis against $S$. aureus recording a mean inhibition diameter and standard error (SE) of $16.33 \pm 0.88 \mathrm{~mm}$, respectively, at $P=0.001$. The 70 bacterial isolates belonged to Bacillus, Paraclostridium, Lysinibacillus, Virgibacillus, and Serratia genera. The study isolated Bacillus wiedmannii (KC75) which is a risk group 2 as well as Serratia marcescens (KMP95) and Bacillus anthracis (KS606) which are risk group 3 organisms. The presence of antibiotic resistance genes Tet A, ${ }^{B l a}$ TEM, StrB, Dfr A, Amp, and FloR genes was confirmed by a polymerase chain reaction. Samples from Kangaru Market recorded a higher (88.57\%) proportion of resistant isolates as compared to isolates from Embu Town (11.43\%). The study confirmed the presence of antibioticresistant bacteria in vended fast food and the soil in Embu Town and Kangaru Market. This study calls for continuous monitoring of bacterial status and hygienic handling of vended food.
\end{abstract}

\section{Introduction}

Urban centers in developing countries face socioeconomic challenges that can adversely affect health standards. Foodborne diseases, particularly those of microbial origin, are a major health problem and contribute to reduced economic growth [1]. Street foods are popular in urban areas because of their availability, convenience, and affordability. However, the hygiene aspects of sales operations are a major source of concern for the health of both handlers and consumers [2]. Running surface water, inadequate sanitation, and the disposal of untreated wastewater provide an environment conducive to the growth of pathogenic microbes. Besides, street foods are prone to cross-contamination due to lack of personal hygiene, sharing of contaminated utensils, and free movement of flies that sporadically land on the food.

Food and environmental samples are reservoirs of foodborne pathogens such as Listeria monocytogenes, Vibrio parahaemolyticus, Escherichia coli, Salmonella spp., Vibrio cholerae, and Staphylococcus aureus [1,3]. In addition to antibiotic resistance in bacterial pathogens, commensal bacteria and the environment are also reservoirs of antibiotic resistance. The release of animal and human waste into the 
environment results in the subsequent release of antibiotics into water and soil, which encourages further spread of antibiotic resistance [4]. Bacterial resistance to commonly used antibiotics has been associated with enteric pathogens such as E. coli, Salmonella spp., Shigella spp., Klebsiella spp., and $V$. cholerae [5].

A cross-sectional study conducted in 2014-2016 in 22 of Kenya's 47 counties diagnosed 11,033 people with cholera [6]. An outbreak of cholera was reported in Embu County in 2019 [7]. Waterborne diseases in Embu County have been attributed to poor sanitation and contamination of water sources [8]. Despite efforts to report the prevalence of foodand waterborne pathogens in Embu County, data on their susceptibility to antibiotics are scarce, so this study focused on the isolation and characterization of antibiotic-resistant bacteria in food sold in Embu Town and the nearby Kangaru Market.

\section{Materials and Methods}

2.1. Study Site and Sampling. Samples consisting of fried fish, African sausages, roasted meat, smokies, samosa, chips (potato fries), vegetable salads, and soil were purposively collected from 176 out of the 324 licensed food vendors in the densely populated Central Business District of Embu Town and the nearby Kangaru Market (latitude: $0^{\circ} 31^{\prime} 58.80^{\prime \prime} \mathrm{N}$ and longitude: $37^{\circ} 27^{\prime} 0.00^{\prime \prime}$ ) (Figure 1). Samples were collected aseptically and transported to the $\mathrm{Mi}$ crobiology Laboratory at the University of Embu where they were stored in a refrigerator at $4^{\circ} \mathrm{C}$. Treated water from Embu Water and Sanitation Company (EWASCO) was also sampled.

\subsection{Preparation of Inoculum and Subculturing. The samples} were incubated in eight selective media, namely, MacConkey agar, Salmonella Shigella agar, brain-heart infusion agar, phenylalanine agar, Pseudomonas fluorescens agar, brilliant green agar, eosin ethidium bromide agar, and deoxycholate agar. The standard cultivation method for bacterial food contaminants isolation was carried out as recommended by the International Organization for Standardization (ISO) 6579 [9]. The plates were incubated at $37^{\circ} \mathrm{C}$ for 24 hours, in an inverted position, after which single colonies were subcultured on fresh media.

\subsection{Characterization and Identification of Bacterial Isolates.}

The isolates were characterized as described in [10]. The ability of the isolates to ferment sugars, utilize citrate, and produce catalase enzyme and phenyl-pyruvic acid was determined as previously described $[11,12]$. The isolates were screened for their ability to produce amylase, protease, cellulase, and esterase using the methods described in $[13,14]$. Probable identification of the isolates was done using Bergey's manual of determinative bacteriology and confirmed by partial sequencing of the $16 \mathrm{~S}$ rRNA gene of some isolates.
2.4. Antibiotic Susceptibility Assay. The Kirby Bauer disc diffusion method using Mueller-Hinton agar was used for antimicrobial susceptibility testing [15]. Antimicrobial discs with amoxicillin $(20 \mu \mathrm{g})$, cefotaxime $(30 \mu \mathrm{g})$, gentamicin $(10 \mu \mathrm{g})$, streptomycin $(10 \mu \mathrm{g})$, tetracycline $(30 \mu \mathrm{g})$, nalidixic acid $(30 \mu \mathrm{g})$, trimethoprim + sulphamethoxazole $(1.25 / 23.75 \mu \mathrm{g})$, and chloramphenicol $(30 \mu \mathrm{g})$ were used. Klebsiella pneumoniae 1792880 and $E$. coli 35218 were used as quality control strains. Bacterial suspensions of pure isolates were prepared based on 0.5 McFarland Turbidity Standard. The plates were incubated in an inverted position at $37^{\circ} \mathrm{C}$ for $24 \mathrm{hrs}$. The diameter of the zones of inhibition (in millimeters) was measured and interpreted as either susceptible, intermediate, or resistant according to the Guidelines of CLSI [16].

2.5. Antibiosis Test. Standardized bacterial suspensions were prepared based on $0.5 \mathrm{McF}$ arland turbidity [17]. The antibiosis test was performed as previously described [18]. The isolates were incubated at $37^{\circ} \mathrm{C}$ and monitored routinely after every 24 hours for two days. The diameter of the zones of inhibition was recorded. The antibiotic-resistant bacteria were tested for antibiosis against six laboratory bacteria strains, namely, Staphylococcus aureus ATCC 1026, Klebsiella pyrope 1792880, E. coli ATCC 35218, Streptococcus pneumoniae ATCC 49619, S. aureus BAA 976, and K. pneumoniae 1792880.

2.6. DNA Extraction from Antibiotic-Resistant Bacteria. The bacterial isolates were grown overnight in Luria Broth for 24 hours, and DNA was extracted using the phenolchloroform method with some modifications [19]. To isolate plasmid DNA, bacterial isolates were grown overnight in Luria Broth. Plasmids were extracted using a protocol modified from [20].

2.7. Partial Sequencing of Bacterial 16S rRNA Gene. The 16S rRNA gene sequence was amplified by PCR using bacterial universal primers $8 \mathrm{~F}\left(5^{\prime}\right.$-AGCTTTGATCCTGGCTCAG-3') and 1492R ( $5^{\prime}$-CGGCTACCTTGTTACGACTT- $\left.3^{\prime}\right)$ for all selected bacterial isolates according to [21] using PhusionHigh fidelity PCR Kit (New England and Biolabs Inc). The PCR amplicons were checked by gel electrophoresis, labeled, and shipped to Inqaba Biotech African's Genomics Company in South Africa for sequencing. The sequence outputs were edited, analyzed, and deposited in GenBank for assignment of accession numbers.

2.8. PCR Amplification of Antibiotic Resistance Genes. Genes encoding resistance to tetracycline, streptomycin, and beta-lactam antibiotics such as ampicillin, chloramphenicol, and trimethoprim were detected using the primer specific PCR approach (Table 1) as described in [22]. The presence of a target gene was confirmed by the presence of a band on the gel.

2.9. Data Analysis. Data on antibiosis, extracellular enzymatic activity, and antibiotic sensitivity were recorded in excel sheets. The general linear model (PROC GLM) procedure of SAS software version 9.1 (SAS Institute, 


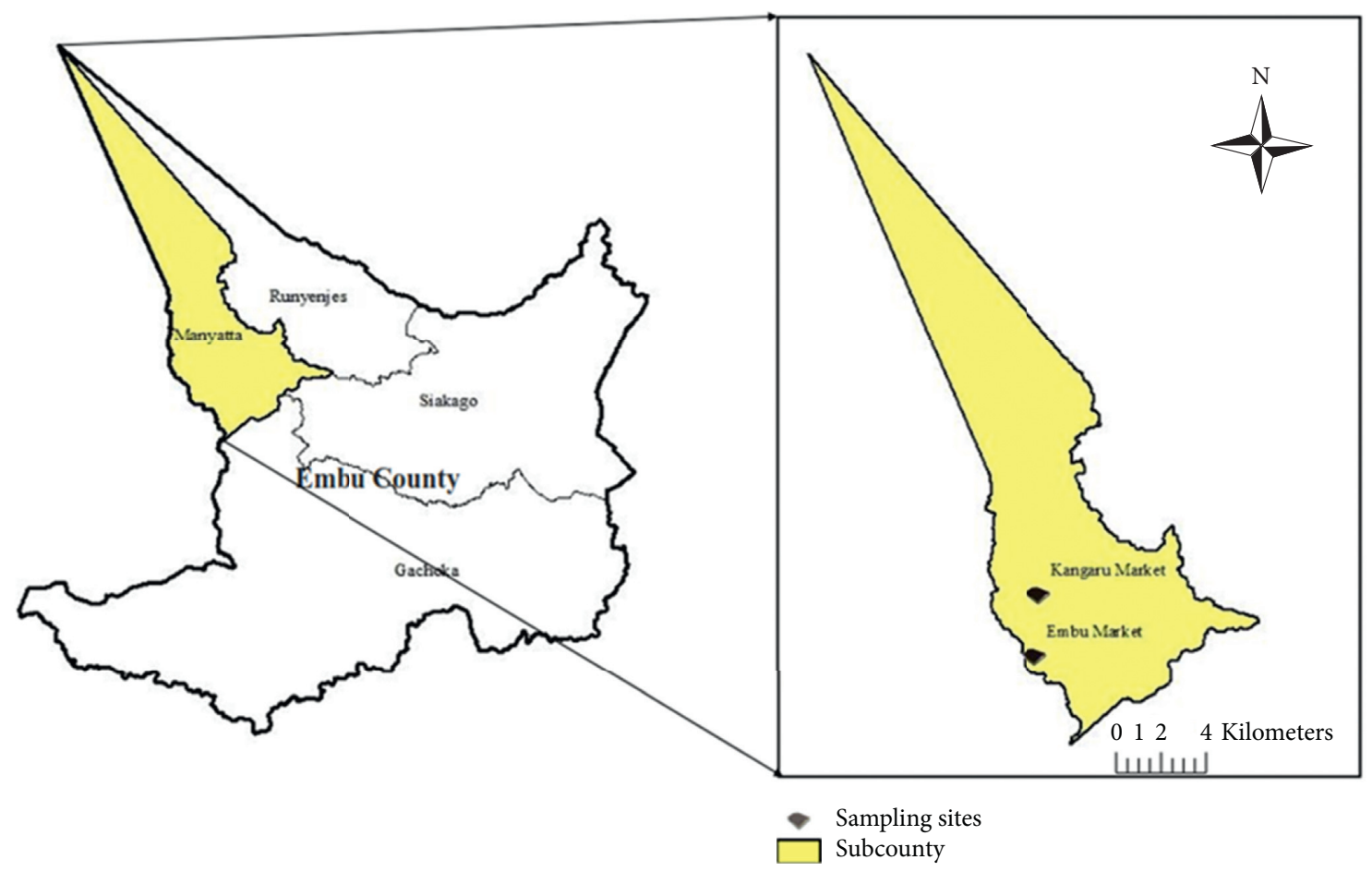

Figure 1: Sampling site in Embu County.

TABLE 1: List of primers for antibiotic resistance genes.

\begin{tabular}{|c|c|c|c|c|c|}
\hline Primer & Sequence $\left(5^{\prime}\right.$ to $\left.3^{\prime}\right)$ & Target gene & Amplicon size (bp) & Annealing temperature $\left({ }^{\circ} \mathrm{C}\right)$ & Resistance to antibiotics \\
\hline $\begin{array}{l}\text { Flor-F } \\
\text { Flor-R }\end{array}$ & $\begin{array}{c}\text { TTATCTCCCTGTCGTTCCAGCG } \\
\text { CCTATGAGCACACGGGGAGC }\end{array}$ & FloR & 450 & 57.5 & Chloramphenicol \\
\hline $\begin{array}{l}\text { strB-F } \\
\text { strB-R }\end{array}$ & $\begin{array}{l}\text { GGCACCCATAAGCGTACGCC } \\
\text { TGCCGAGCACGGCGACTACC }\end{array}$ & Str B & 400 & 61 & Streptomycin \\
\hline $\begin{array}{l}\text { DfrA1-F } \\
\text { DfrA1-R }\end{array}$ & $\begin{array}{l}\text { CGAAGAATGGAGTTATCGGG } \\
\text { TGCTGGGGATTTCAGGAAAG }\end{array}$ & $D f r$ A & 200 & 55 & Trimethoprim \\
\hline $\begin{array}{l}\text { TEM-F } \\
\text { TEM-R }\end{array}$ & $\begin{array}{l}\text { GCGGTATCATTGCAGCACTG } \\
\text { TGCTTAATCAGTGAGGCACC }\end{array}$ & ${ }^{B l a} \mathrm{TEM}$ & 1000 & 55 & Beta-lactam \\
\hline $\begin{array}{l}\text { Amp-F } \\
\text { Amp-R } \\
\end{array}$ & $\begin{array}{l}\text { ATGCACACGCTGATCGGATT } \\
\text { GCGGACGCAGACTTCACTAA }\end{array}$ & $A m p$ & 268 & 65 & Ampicillin \\
\hline $\begin{array}{l}\text { Tet-F } \\
\text { Tet-R }\end{array}$ & $\begin{array}{l}\text { AACCGGCATTGAGAGCATCA } \\
\text { TTGTCTCCTCTCCCTTGGCT }\end{array}$ & Tet A & 699 & 60 & Tetracycline \\
\hline
\end{tabular}

Cary, NC) was used to perform analysis of variance (ANOVA). Tukey's honest significant difference (HSD) test was used to compare and separate the means of the diameter of zones of inhibition. Correlation profiles of zones of clearance on different substrates and zones of inhibition were visualized as a heatmap generated by a hierarchical clustering $R$ script using $R$ version 3.3.1 software. The genetic affiliation of the sequenced genes of the isolates was deduced from a phylogenetic tree generated using MEGA X. The analysis involved 20 nucleotide sequences. The evolutionary distances were computed using the maximum composite likelihood method and were recorded as units of the number of base substitutions.

\section{Results}

3.1. Effect of Antibiotics on Bacteria Isolated from Food and Soil. Antibiotic sensitivity test assays revealed that 70 out of 345 bacterial isolates were resistant to at least three (multiple resistant) antibiotics that were tested. Isolate KMP253 affiliated to Salmonella spp., TF152 affiliated to E. coli, and KS120 affiliated to Bacillus subtilis were resistant to all the antibiotics that were tested and were all isolated from Kangaru Market (Table 2). Hierarchical clustering showed that trimethoprim + sulphamethoxazole was the most effective antibiotic, while chloramphenicol was the least effective antibiotic. Isolate KVS120 was resistant to the highest number of antibiotics (Figure 2). The occurrence of 
TABLe 2: Antibiotic resistance of bacteria isolated from vended food and the soil in Embu Town and Kangaru Market in 2018.

\begin{tabular}{|c|c|c|c|c|c|c|c|c|c|c|c|c|}
\hline Isolate & Probable identity & Location & Sample & Strep & Genta & Amox & Tetra & Chlo & $\begin{array}{l}\text { Tri- } \\
\text { sul }\end{array}$ & Cefotax & Nalid & $\begin{array}{l}\text { Resistant to } \\
\text { antibiotics } \\
(n)\end{array}$ \\
\hline KS7 & Providencia spp. & Kangaru & Soil & $\mathrm{R}$ & $\mathrm{I}$ & $\mathrm{R}$ & $\mathrm{R}$ & I & $\mathrm{I}$ & I & $\mathrm{R}$ & 4 \\
\hline KF27 & Shigella spp. & Kangaru & Fish & $S$ & $\mathrm{R}$ & $\mathrm{R}$ & $\mathrm{R}$ & $\mathrm{R}$ & $\mathrm{R}$ & $\mathrm{R}$ & $\mathrm{R}$ & 7 \\
\hline KRM34 & E. coli & Kangaru & Roasted meat & S & I & $\mathrm{R}$ & $\mathrm{R}$ & $\mathrm{R}$ & I & $\mathrm{R}$ & $\mathrm{R}$ & 5 \\
\hline KFR38 & Bacillus pacificus & Kangaru & Fruit & I & $S$ & $\mathrm{R}$ & $\mathrm{R}$ & $\mathrm{R}$ & $\mathrm{R}$ & $\mathrm{R}$ & $\mathrm{R}$ & 6 \\
\hline KFR42 & Bacillus amyloliquefaciens & Kangaru & Fruit & I & $\mathrm{R}$ & $\mathrm{R}$ & $\mathrm{R}$ & $\mathrm{R}$ & I & $\mathrm{R}$ & $\mathrm{R}$ & 6 \\
\hline KMP45 & Pseudomonas spp. & Kangaru & $\begin{array}{l}\text { African } \\
\text { sausage }\end{array}$ & $\mathrm{R}$ & I & I & I & $\mathrm{R}$ & I & $\mathrm{R}$ & S & 3 \\
\hline KFR52 & Bacillus megaterium & Kangaru & Fruit & I & I & $\mathrm{R}$ & $\mathrm{R}$ & $\mathrm{R}$ & I & $\mathrm{R}$ & $\mathrm{R}$ & 5 \\
\hline KSM63 & Shigella sonnei & Kangaru & Smokies & $\mathrm{R}$ & S & I & $\mathrm{R}$ & I & I & $\mathrm{R}$ & I & 3 \\
\hline KS65 & Bacillus wiedmannii & Kangaru & Soil & I & S & $\mathrm{R}$ & S & $\mathrm{R}$ & I & $\mathrm{R}$ & $S$ & 3 \\
\hline KC67 & Pseudomonas aeruginosa & Kangaru & Chips & S & S & $\mathrm{R}$ & $\mathrm{R}$ & I & S & $\mathrm{R}$ & $\mathrm{R}$ & 4 \\
\hline KVS68 & Enterobacter spp. & Kangaru & $\begin{array}{l}\text { Vegetable } \\
\text { salad }\end{array}$ & S & $\mathrm{R}$ & $\mathrm{R}$ & $\mathrm{R}$ & $\mathrm{R}$ & $\mathrm{R}$ & S & S & 5 \\
\hline KVS75 & Bacillus wiedmannii & Kangaru & $\begin{array}{l}\text { Vegetable } \\
\text { salad }\end{array}$ & I & S & S & $\mathrm{R}$ & $\mathrm{R}$ & S & $\mathrm{R}$ & S & 3 \\
\hline TVS81 & Bacillus thuringiensis & Kangaru & $\begin{array}{l}\text { Vegetable } \\
\text { salad }\end{array}$ & S & S & $\mathrm{R}$ & $\mathrm{R}$ & $\mathrm{R}$ & I & $\mathrm{R}$ & $\mathrm{R}$ & 5 \\
\hline KVS82 & Serratia marcescens & Kangaru & $\begin{array}{l}\text { Vegetable } \\
\text { salad }\end{array}$ & S & $\mathrm{R}$ & $\mathrm{R}$ & S & $\mathrm{R}$ & $S$ & $\mathrm{R}$ & $\mathrm{R}$ & 5 \\
\hline KVS85 & Serratia marcescens & Kangaru & $\begin{array}{l}\text { Vegetable } \\
\text { salad }\end{array}$ & S & S & $\mathrm{R}$ & S & $\mathrm{R}$ & $S$ & I & $\mathrm{R}$ & 3 \\
\hline KMP95 & Serratia marcescens & Kangaru & $\begin{array}{l}\text { African } \\
\text { sausage }\end{array}$ & $\mathrm{R}$ & S & I & $\mathrm{R}$ & $\mathrm{R}$ & $\mathrm{R}$ & $\mathrm{R}$ & $\mathrm{R}$ & 6 \\
\hline KF101 & Bacillus velezensis & Kangaru & Fish & $S$ & $S$ & $S$ & $\mathrm{R}$ & $\mathrm{R}$ & S & $\mathrm{R}$ & $\mathrm{R}$ & 4 \\
\hline KS104 & Virgibacillus phasianinus & Kangaru & Soil & S & $\mathrm{R}$ & I & S & $\mathrm{R}$ & S & S & $\mathrm{R}$ & 3 \\
\hline KS109 & $\begin{array}{c}\text { Lysinibacillus } \\
\text { parviboronicapiens }\end{array}$ & Kangaru & Soil & $\mathrm{R}$ & $\mathrm{R}$ & $\mathrm{R}$ & $\mathrm{R}$ & $\mathrm{R}$ & I & $\mathrm{R}$ & $\mathrm{R}$ & 7 \\
\hline KS116 & Providencia spp. & Kangaru & Soil & $\mathrm{R}$ & $\mathrm{R}$ & $\mathrm{R}$ & $\mathrm{R}$ & $\mathrm{R}$ & $\mathrm{R}$ & $\mathrm{R}$ & $\mathrm{R}$ & 8 \\
\hline KVS120 & Bacillus subtilis & Kangaru & $\begin{array}{l}\text { Vegetable } \\
\text { salad }\end{array}$ & $\mathrm{R}$ & $\mathrm{R}$ & $\mathrm{R}$ & $\mathrm{R}$ & $\mathrm{R}$ & $\mathrm{R}$ & $\mathrm{R}$ & $\mathrm{R}$ & 8 \\
\hline KC122 & Pseudomonas spp. & Kangaru & Chips & $S$ & $S$ & I & I & $\mathrm{R}$ & S & $\mathrm{R}$ & $\mathrm{R}$ & 3 \\
\hline KFR131 & Pseudomonas spp. & Kangaru & Fruit & $\mathrm{R}$ & I & $\mathrm{R}$ & $\mathrm{R}$ & $\mathrm{R}$ & I & $\mathrm{R}$ & $\mathrm{R}$ & 6 \\
\hline KVS147 & Staphylococcus aureus & Kangaru & $\begin{array}{l}\text { Vegetable } \\
\text { salad }\end{array}$ & $\mathrm{R}$ & $\mathrm{R}$ & $\mathrm{R}$ & S & $\mathrm{R}$ & S & $S$ & $\mathrm{R}$ & 5 \\
\hline KF148 & Shigella spp. & Kangaru & Fish & I & S & $\mathrm{R}$ & S & $\mathrm{R}$ & S & $\mathrm{S}$ & $\mathrm{R}$ & 3 \\
\hline TF152 & E. coli & Town & Fish & $\mathrm{R}$ & $\mathrm{R}$ & $\mathrm{R}$ & $\mathrm{R}$ & $\mathrm{R}$ & $\mathrm{R}$ & $\mathrm{R}$ & $\mathrm{R}$ & 8 \\
\hline KMP159 & Salmonella spp. & Kangaru & $\begin{array}{l}\text { African } \\
\text { sausage }\end{array}$ & I & $\mathrm{R}$ & I & S & $\mathrm{R}$ & S & S & $\mathrm{R}$ & 3 \\
\hline KS160 & Citrobacter spp. & Kangaru & Soil & $\mathrm{R}$ & $\mathrm{R}$ & $\mathrm{R}$ & $\mathrm{R}$ & $\mathrm{R}$ & I & $\mathrm{R}$ & $\mathrm{R}$ & 7 \\
\hline KF169 & Proteus spp. & Kangaru & Fish & $\mathrm{R}$ & $\mathrm{R}$ & $\mathrm{R}$ & S & $\mathrm{R}$ & S & S & $\mathrm{R}$ & 5 \\
\hline KMP188 & Aerobacter aerogenes & Kangaru & $\begin{array}{l}\text { African } \\
\text { sausage }\end{array}$ & $\mathrm{R}$ & $\mathrm{R}$ & $\mathrm{R}$ & S & $\mathrm{R}$ & S & S & $\mathrm{R}$ & 5 \\
\hline KFR200 & Enterobacter spp. & Kangaru & Fruit & $S$ & I & $S$ & $\mathrm{R}$ & $\mathrm{R}$ & I & $\mathrm{R}$ & I & 3 \\
\hline KFR204 & Pseudomonas spp. & Kangaru & Fruit & S & I & $\mathrm{R}$ & $\mathrm{R}$ & $\mathrm{R}$ & I & $\mathrm{R}$ & $\mathrm{R}$ & 5 \\
\hline KVS214 & S. pneumoniae & Kangaru & $\begin{array}{l}\text { Vegetable } \\
\text { salad }\end{array}$ & $\mathrm{R}$ & $\mathrm{R}$ & $\mathrm{R}$ & $\mathrm{R}$ & S & $\mathrm{R}$ & $\mathrm{R}$ & $\mathrm{R}$ & 7 \\
\hline KFR217 & Klebsiella spp. & Kangaru & Fruit & $\mathrm{R}$ & $S$ & $S$ & $\mathrm{R}$ & $\mathrm{R}$ & $S$ & $\mathrm{R}$ & $\mathrm{R}$ & 5 \\
\hline KFR222 & Bacillus mobilis & Kangaru & Fruit & S & S & $\mathrm{R}$ & $\mathrm{R}$ & $\mathrm{R}$ & S & $\mathrm{R}$ & $\mathrm{R}$ & 5 \\
\hline KC231 & S. pneumoniae & Kangaru & Chips & $S$ & $S$ & I & $S$ & $\mathrm{R}$ & $S$ & $\mathrm{R}$ & $\mathrm{R}$ & 3 \\
\hline KFR245 & P. aeruginosa & Kangaru & Fruit & S & S & I & I & $\mathrm{R}$ & I & $\mathrm{R}$ & $\mathrm{R}$ & 3 \\
\hline KF246 & S. pneumoniae & Kangaru & Fish & I & $S$ & I & $\mathrm{R}$ & $\mathrm{R}$ & I & $\mathrm{R}$ & $\mathrm{R}$ & 4 \\
\hline KVS249 & Pseudomonas spp. & Kangaru & $\begin{array}{l}\text { Vegetable } \\
\text { salad }\end{array}$ & I & $\mathrm{R}$ & $\mathrm{R}$ & $\mathrm{R}$ & $\mathrm{R}$ & $\mathrm{R}$ & $\mathrm{R}$ & $\mathrm{R}$ & 7 \\
\hline KMP253 & Salmonella spp. & Kangaru & $\begin{array}{l}\text { African } \\
\text { sausage }\end{array}$ & $\mathrm{R}$ & $\mathrm{R}$ & $\mathrm{R}$ & $\mathrm{R}$ & $\mathrm{R}$ & $\mathrm{R}$ & $\mathrm{R}$ & $\mathrm{R}$ & 8 \\
\hline KS260 & E. coli & Kangaru & Soil & I & $\mathrm{R}$ & $S$ & $\mathrm{R}$ & $\mathrm{R}$ & I & $\mathrm{R}$ & $\mathrm{R}$ & 5 \\
\hline KS267 & Bacillus wiedmannii & Kangaru & Soil & I & $\mathrm{R}$ & $\mathrm{R}$ & $\mathrm{R}$ & $\mathrm{R}$ & $\mathrm{R}$ & $\mathrm{R}$ & $\mathrm{R}$ & 7 \\
\hline
\end{tabular}


TABLE 2: Continued.

\begin{tabular}{|c|c|c|c|c|c|c|c|c|c|c|c|c|}
\hline Isolate & Probable identity & Location & Sample & Strep & Genta & Amox & Tetra & Chlo & $\begin{array}{l}\text { Tri- } \\
\text { sul }\end{array}$ & Cefotax & Nalid & $\begin{array}{c}\text { Resistant to } \\
\text { antibiotics } \\
(n)\end{array}$ \\
\hline KVS271 & Pseudomonas spp. & Kangaru & $\begin{array}{l}\text { Vegetable } \\
\text { salad }\end{array}$ & I & $\mathrm{R}$ & I & $\mathrm{R}$ & $\mathrm{R}$ & I & $\mathrm{R}$ & $\mathrm{R}$ & 5 \\
\hline KFR285 & Hafnia spp. & Kangaru & Fruit & S & $\mathrm{R}$ & I & $S$ & $\mathrm{R}$ & $S$ & S & $\mathrm{R}$ & 3 \\
\hline KFR286 & Pseudomonas spp. & Kangaru & Fruit & I & $S$ & $\mathrm{R}$ & $\mathrm{R}$ & $\mathrm{R}$ & $S$ & $\mathrm{R}$ & $\mathrm{R}$ & 5 \\
\hline KFR301 & Salmonella spp. & Kangaru & Fruit & I & $\mathrm{R}$ & $\mathrm{R}$ & $\mathrm{R}$ & $\mathrm{R}$ & $\mathrm{R}$ & $\mathrm{R}$ & $\mathrm{R}$ & 7 \\
\hline KF317 & Streptococcus pneumoniae & Kangaru & Fish & $\mathrm{R}$ & S & I & $\mathrm{R}$ & $\mathrm{R}$ & I & $\mathrm{R}$ & $\mathrm{R}$ & 5 \\
\hline KMP321 & Bacillus wiedmannii & Kangaru & $\begin{array}{l}\text { African } \\
\text { sausage }\end{array}$ & $\mathrm{R}$ & $\mathrm{R}$ & $\mathrm{R}$ & $\mathrm{R}$ & $\mathrm{R}$ & I & $\mathrm{R}$ & $\mathrm{R}$ & 7 \\
\hline TFR329 & Bacillus proteolyticus & Town & Fruit & S & $\mathrm{R}$ & $\mathrm{R}$ & $\mathrm{R}$ & $\mathrm{R}$ & $\mathrm{R}$ & $\mathrm{R}$ & $\mathrm{R}$ & 7 \\
\hline KVS330 & Citrobacter freundii & Kangaru & $\begin{array}{c}\text { Vegetable } \\
\text { salad }\end{array}$ & $S$ & $\mathrm{R}$ & I & $\mathrm{R}$ & $\mathrm{R}$ & $\mathrm{R}$ & $\mathrm{R}$ & I & 5 \\
\hline KMP337 & Salmonella spp. & Kangaru & $\begin{array}{l}\text { African } \\
\text { sausage }\end{array}$ & I & I & I & $\mathrm{R}$ & $\mathrm{R}$ & I & $\mathrm{R}$ & $\mathrm{R}$ & 4 \\
\hline KRM349 & Pseudomonas spp. & Kangaru & Roasted meat & I & I & $\mathrm{R}$ & $\mathrm{R}$ & $\mathrm{R}$ & I & $\mathrm{R}$ & $\mathrm{R}$ & 5 \\
\hline KS376 & E. coli & Kangaru & Soil & S & $\mathrm{R}$ & $\mathrm{R}$ & $\mathrm{R}$ & $\mathrm{R}$ & $\mathrm{R}$ & $\mathrm{R}$ & $\mathrm{R}$ & 7 \\
\hline TS378 & Salmonella spp. & Town & Soil & I & $\mathrm{R}$ & $\mathrm{R}$ & $\mathrm{R}$ & $\mathrm{R}$ & I & $\mathrm{R}$ & $\mathrm{R}$ & 6 \\
\hline TS380 & Pseudomonas spp. & Town & Soil & $\mathrm{R}$ & $S$ & $\mathrm{R}$ & $\mathrm{R}$ & $\mathrm{R}$ & $\mathrm{R}$ & $\mathrm{R}$ & $\mathrm{R}$ & 7 \\
\hline KF381 & Micrococcus spp. & Kangaru & Fish & $S$ & $\mathrm{R}$ & $\mathrm{R}$ & $\mathrm{R}$ & $\mathrm{R}$ & $\mathrm{R}$ & $\mathrm{R}$ & $\mathrm{R}$ & 7 \\
\hline KF389 & Morganella spp. & Kangaru & Fish & I & S & $\mathrm{R}$ & I & $\mathrm{R}$ & I & $\mathrm{R}$ & S & 3 \\
\hline KF391 & Serratia marcescens & Kangaru & Fish & $\mathrm{R}$ & $\mathrm{R}$ & $\mathrm{R}$ & $\mathrm{R}$ & $\mathrm{R}$ & $\mathrm{R}$ & $\mathrm{R}$ & $\mathrm{S}$ & 7 \\
\hline KF393 & Yersinia spp. & Kangaru & Fish & S & $\mathrm{R}$ & $\mathrm{R}$ & $\mathrm{R}$ & $\mathrm{R}$ & I & $\mathrm{R}$ & $\mathrm{R}$ & 6 \\
\hline KF395 & Pseudomonas aeruginosa & Kangaru & Fish & S & I & $\mathrm{R}$ & $\mathrm{R}$ & $\mathrm{R}$ & $S$ & $\mathrm{R}$ & $\mathrm{R}$ & 5 \\
\hline KF399 & Salmonella enterica & Kangaru & Fish & I & $\mathrm{R}$ & $\mathrm{R}$ & $\mathrm{R}$ & $\mathrm{R}$ & $\mathrm{R}$ & $\mathrm{R}$ & $\mathrm{R}$ & 7 \\
\hline TS427 & Pseudomonas spp. & Town & Soil & I & $\mathrm{R}$ & $\mathrm{R}$ & $\mathrm{R}$ & $\mathrm{R}$ & I & $\mathrm{R}$ & $S$ & 5 \\
\hline KC430 & Pseudomonas spp. & Kangaru & Chips & I & $\mathrm{S}$ & $\mathrm{R}$ & $\mathrm{R}$ & $\mathrm{R}$ & $\mathrm{R}$ & $\mathrm{R}$ & $\mathrm{R}$ & 6 \\
\hline KRM435 & Bacillus wiedmannii & Kangaru & Roasted meat & I & S & $\mathrm{R}$ & $\mathrm{R}$ & I & S & $\mathrm{R}$ & $\mathrm{R}$ & 4 \\
\hline KF463 & Bacillus anthracis & Kangaru & Fish & $\mathrm{R}$ & $\mathrm{R}$ & $\mathrm{R}$ & $\mathrm{R}$ & $\mathrm{R}$ & I & $\mathrm{R}$ & $\mathrm{R}$ & 7 \\
\hline TS472 & Bacillus cereus & Town & Soil & S & $\mathrm{R}$ & $\mathrm{R}$ & $\mathrm{R}$ & $\mathrm{R}$ & $\mathrm{R}$ & $\mathrm{R}$ & $\mathrm{R}$ & 7 \\
\hline TS572 & Bacillus subtilis & Town & Soil & S & S & $\mathrm{R}$ & $\mathrm{R}$ & $\mathrm{R}$ & I & $\mathrm{R}$ & $\mathrm{R}$ & 5 \\
\hline KS585 & Enterococcus faecalis & Kangaru & Soil & $\mathrm{R}$ & $\mathrm{R}$ & $\mathrm{R}$ & $\mathrm{R}$ & $\mathrm{R}$ & $\mathrm{R}$ & $S$ & $\mathrm{R}$ & 7 \\
\hline KS606 & Bacillus anthracis & Kangaru & Soil & $\mathrm{R}$ & I & $\mathrm{R}$ & $\mathrm{R}$ & $\mathrm{R}$ & I & $\mathrm{R}$ & $\mathrm{S}$ & 5 \\
\hline TS621 & Proteus spp. & Town & Soil & S & $\mathrm{R}$ & $\mathrm{R}$ & $\mathrm{S}$ & $\mathrm{R}$ & I & $\mathrm{R}$ & $\mathrm{R}$ & 5 \\
\hline
\end{tabular}

Strep, streptomycin; Genta, gentamycin; Amox, amoxicillin; tetra, tetracycline; Chlo, chloramphenicol; Tri-Sul, trimethoprim + sulphamethoxazole; Cefotax, cefotaxime; Nalid, nalidixic acid.

antibiotic-resistant bacteria varied among different food samples. Smokies from both sites had the lowest number of resistant bacterial isolates (1.43\%) compared to the other food samples. Fish sold in Kangaru Market had the highest proportion of resistant isolates (18.57\%), as shown in Figure 3. No bacteria were recovered from treated water provided by EWASCO.

3.2. Detection of Antibiotic-Resistant Genes. Antibiotic resistance genes were detected in 36 out of the 70 antibioticresistant bacterial isolates (Table 3 ). The antibiotic resistance genes ${ }^{B l a}$ TEM and $D f r$ A were frequently detected (Figures 4(a) and 4(b)). Resistance genes were detected at a higher frequency in bacteria isolated from Kangaru Market compared to Embu Town.
3.3. Antibiosis Activity. Eleven out of the 70 antibiotic-resistant bacterial isolates inhibited the growth of at least one of the following laboratory strains of bacteria: S. aureus ATCC 1026, E. coli ATCC 35218, and K. pneumoniae 1792880. Isolate KFR38 affiliated to B. pacificus inhibited two laboratory strains and exhibited large zones of inhibition (Table 4). None of the antibiotic-resistant isolates was able to inhibit the growth of K. pyrope 1792880, S. pneumoniae ATCC 49619, or S. aureus BAA 976.

3.4. Risk Groups of Antibiotic-Resistant Bacterial Isolates. The 14 antibiotic-resistant bacteria that were successfully sequenced belonged to risk groups 1, 2, and 3. Isolate KS606 affiliated to B. anthracis and isolate KMP95 affiliated to $S$. marcescens which are risk group 3 organisms that were isolated from food sold in Kangaru Market (Table 5). 

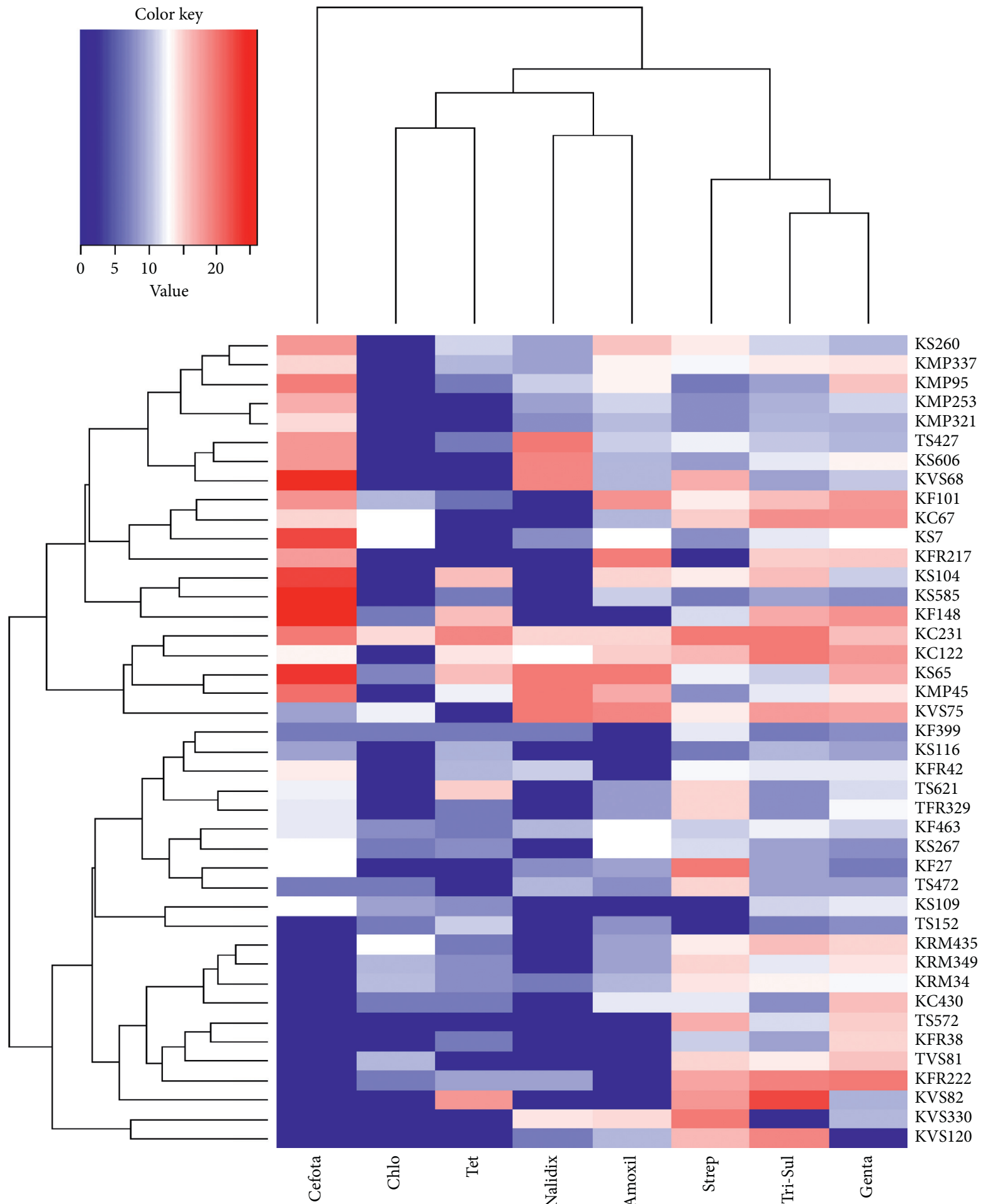

Figure 2: Activity of eight antibiotics against antibiotic-resistant bacteria isolated from vended food and soil in Embu Town and Kangaru Market. Strep, streptomycin; Genta, gentamycin; Amox, amoxicillin; tetra, tetracycline; Chlo, chloramphenicol; Tri-Sul, trimethoprim + sulphamethoxazole; Cefotax, cefotaxime; Nalid, nalidixic acid.

3.5. Substrate Utilization by Antibiotic-Resistant Bacteria. The antibiotic-resistant bacterial isolates produced different extracellular enzymes, as shown by the zones of clearance on different substrates. Isolates KF148, KF317, KS116, KS7, and
KVS249 exhibited the largest zones of clearance with an average diameter and standard error (SE) of $14.67 \pm 0.33 \mathrm{~mm}$, $14.00 \pm 0.58 \mathrm{~mm}, \quad 14.00 \pm 0.58 \mathrm{~mm}, \quad 15.00 \pm 0.00 \mathrm{~mm}$, and $14.67 \pm 0.33 \mathrm{~mm}$, respectively. B. anthracis (KS606) recorded the 


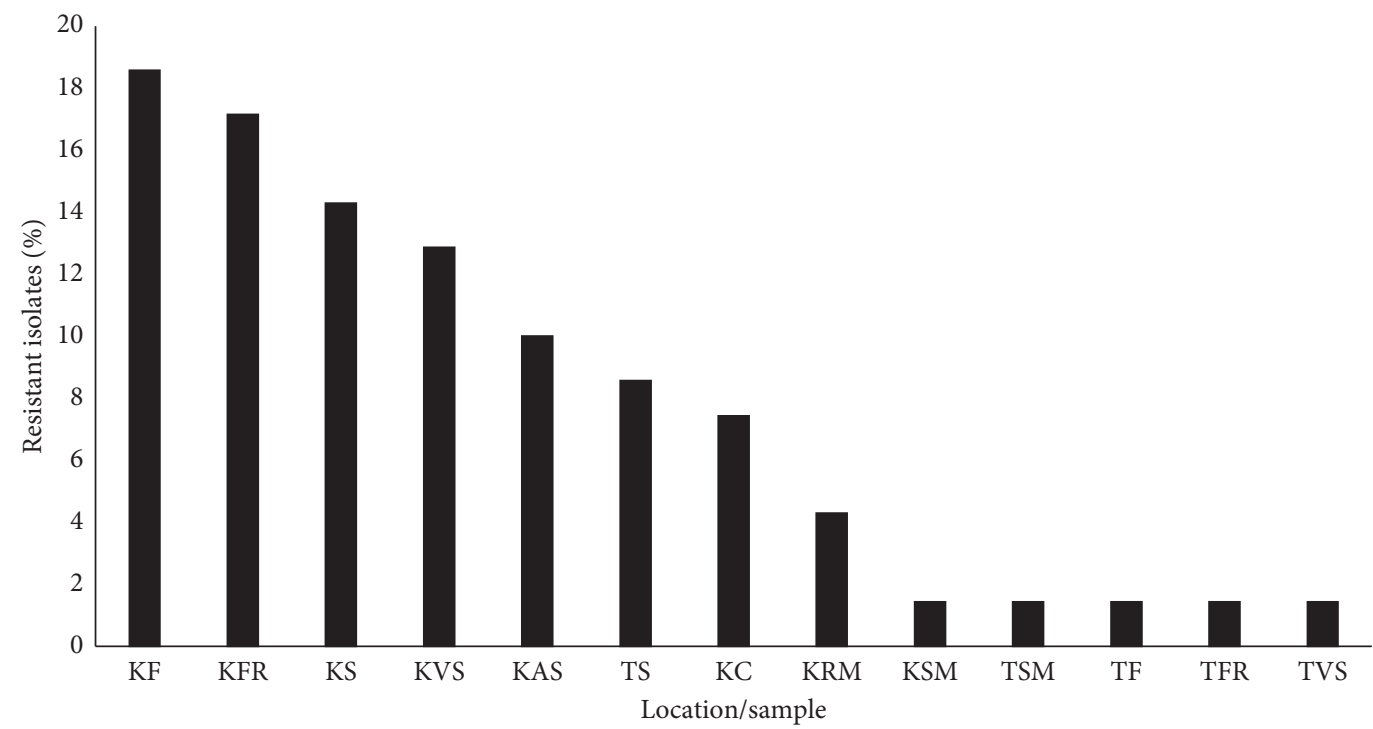

Figure 3: Proportion of antibiotic-resistant bacteria from food vended in Embu Town and Kangaru Market. KAS, Kangaru African sausage; KRM, Kangaru roasted meat; KF, Kangaru fish; TVS, town vegetable salad; KVS, Kangaru vegetable salad; KC, Kangaru chips; TS, town soil; KSM, Kangaru smokies; KFR, Kangaru fruit; KS, Kangaru soil; TFR, town fruit; TF, town fruits; KAS, Kangaru African sausage.

Table 3: Detection of antibiotic resistance genes in bacteria isolated from vended food and soil in Embu Town and Kangaru Market.

\begin{tabular}{|c|c|c|c|c|c|c|c|c|c|}
\hline \multirow{2}{*}{ Isolate number } & \multirow{2}{*}{ Location } & \multirow{2}{*}{ Sample } & \multirow{2}{*}{ Probable identity } & \multicolumn{6}{|c|}{ Detected antibiotic resistance genes } \\
\hline & & & & $D f r$ A & Str B & Tet A & ${ }^{B l a}$ TEM & $A m p$ & FloR \\
\hline KFR222 & Kangaru & Fruit & B. mobilis & - & - & - & + & - & - \\
\hline KF169 & Kangaru & Fish & Proteus spp. & - & - & - & + & - & - \\
\hline KF463 & Kangaru & Fish & B. anthracis & - & + & - & + & - & - \\
\hline KMP253 & Kangaru & African sausage & Salmonella spp. & - & + & - & + & - & - \\
\hline KFR285 & Kangaru & Fruit & Hafnia spp. & - & - & + & + & - & - \\
\hline KF27 & Kangaru & Fish & Shigella spp. & - & - & - & + & - & + \\
\hline KMP188 & Kangaru & African sausage & A. aerogenes & - & - & - & + & + & - \\
\hline KF148 & Kangaru & Fish & Shigella spp. & - & - & - & + & - & + \\
\hline KC67 & Kangaru & Chips & P. aeruginosa & - & - & - & + & - & - \\
\hline TS572 & Town & Soil & B. subtilis & - & + & - & + & - & + \\
\hline KMP95 & Kangaru & African sausage & S. marcescens & + & - & - & - & - & + \\
\hline TFR329 & Town & Fruit & B. proteolyticus & + & - & + & - & - & - \\
\hline KFR301 & Kangaru & Fruit & Salmonella spp. & + & - & - & - & + & - \\
\hline KVS330 & Kangaru & Vegetable salad & E. coli & + & - & + & - & - & - \\
\hline TS380 & Town & Soil & Salmonella spp. & + & - & - & - & - & - \\
\hline TVS81 & Town & Vegetable salad & B. thuringiensis & + & - & - & - & + & - \\
\hline KF391 & Kangaru & Fish & Morganella spp. & + & + & + & - & - & - \\
\hline KS160 & Kangaru & Soil & Citrobacter spp. & - & + & - & - & - & - \\
\hline KF169 & Kangaru & Fish & Proteus spp. & - & + & - & - & + & - \\
\hline KF317 & Kangaru & Fish & E. faecalis & - & + & - & - & - & - \\
\hline KFR131 & Kangaru & Fruit & Pseudomonas spp. & - & + & - & - & - & - \\
\hline KFR38 & Kangaru & Fruit & B. pacificus & - & + & - & - & - & - \\
\hline KVS85 & Kangaru & Vegetable salad & S. marcescens & - & - & - & - & + & - \\
\hline KFR204 & Kangaru & Fruit & Pseudomonas spp. & - & - & - & - & + & - \\
\hline KS109 & Kangaru & Soil & L. parviboronicapiens & - & - & - & - & + & - \\
\hline KVS214 & Kangaru & Vegetable salad & S. pneumoniae & - & - & - & - & + & - \\
\hline KS585 & Kangaru & Soil & B. toyonensis & - & - & - & - & + & + \\
\hline KF399 & Kangaru & Fish & P. aeruginosa & - & - & + & - & + & - \\
\hline KS267 & Kangaru & Soil & B. wiedmannii & - & - & - & - & + & - \\
\hline KS116 & Kangaru & Soil & Providencia spp. & - & - & - & - & + & - \\
\hline KC122 & Kangaru & Chips & Pseudomonas spp. & - & - & - & - & - & + \\
\hline KFR245 & Kangaru & Fruit & $P$. aeruginosa & - & - & - & - & - & + \\
\hline KS376 & Kangaru & Soil & Pseudomonas spp. & - & - & - & - & - & + \\
\hline KVS249 & Kangaru & Vegetable salad & Pseudomonas spp. & - & - & + & - & - & - \\
\hline KS606 & Kangaru & Soil & B. anthracis & - & - & + & - & - & - \\
\hline KF393 & Kangaru & Fish & S. marcescens & - & - & + & - & - & - \\
\hline
\end{tabular}

+ indicates the presence of the resistance genes following amplification by PCR; - indicates the absence of the target resistance genes following amplification by PCR. 


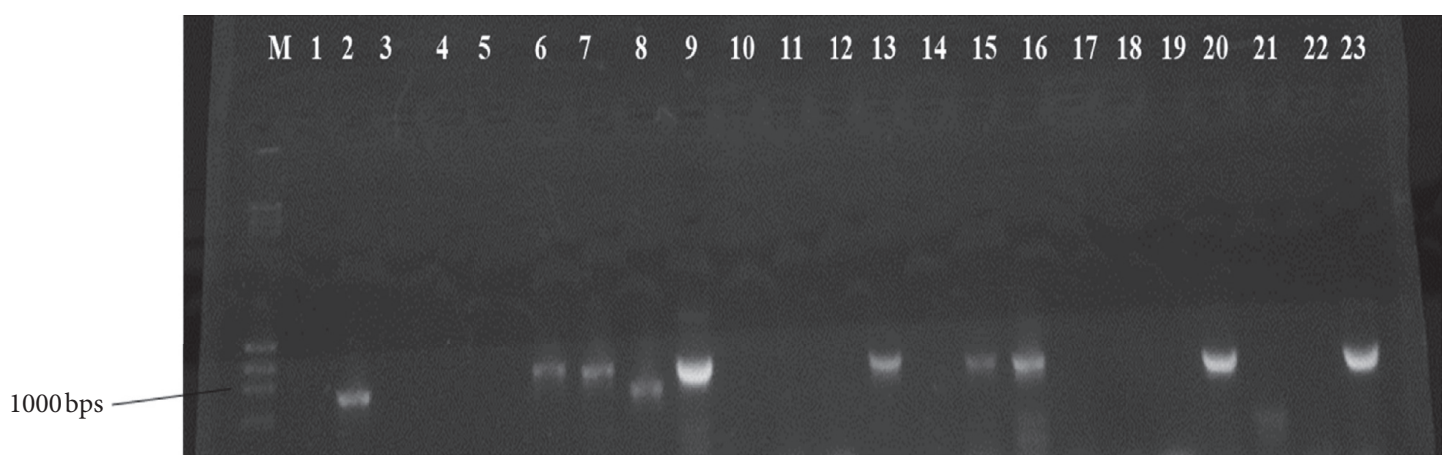

(a)

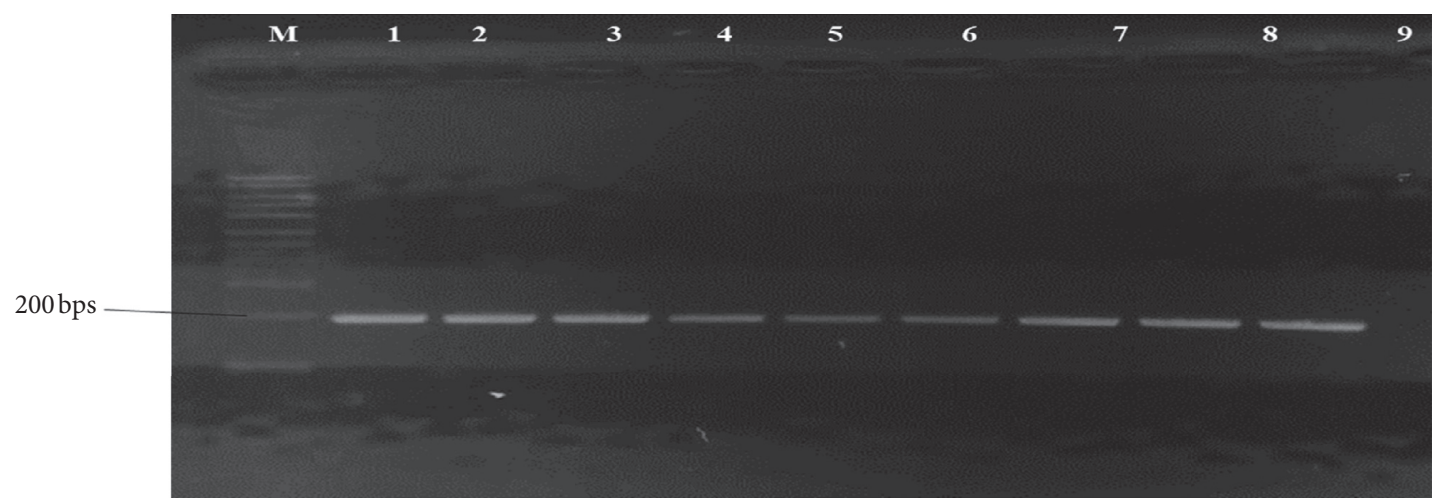

(b)

Figure 4: (a) Antibiotic resistance gene ${ }^{B l a}$ TEM detected in bacterial isolates that were resistant to beta-lactam antibiotics. $M$ is the molecular marker; lane 2, isolate KFR222; 6, KF169; 7, KF463, 8, KMP253; 9, KFR285; 13, KF27; 15, KMP188; 16, KF148; 20, KC67; 23, TS572. The band size is in base pairs. (b) Antibiotic resistance gene $D f r$ A detected in isolates that were resistant to trimethoprim. $M$ is the molecular marker; lane 1, isolate KMP95; 2, TFR329; 3, KFR301; 4, KVS330; 5, TS380; 7, TVS81; 8, KF27; 9, KF391; 10, KF391. The band size is in base pairs.

largest zone of clearance on cellulose, while KC430 affiliated to Pseudomonas spp. had the largest zone of precipitation on the tween-20 substrate.

3.6. Amplification of $16 S$ rRNA Genes and Phylogenetic Analysis. The 16S rRNA gene was successfully amplified in 47 isolates out of the 70 antibiotic-resistant bacterial isolates (Figure 5).

Phylogenetic analysis of successfully sequenced bacterial isolates revealed that the isolates belonged to five genera, namely, Bacillus, Paraclostridium, Lysinibacillus, Virgibacillus, and Serratia with the similarity of above $90 \%$. The Bacillus clade had the highest number of successfully amplified bacterial isolates distributed in nine subclades with bootstrap values of 98 (Figure 6).

\section{Discussion}

The present study isolated 70 bacterial isolates that were resistant to at least three antibiotics from vended foods and the soil in Embu Town and Kangaru Market. Higher levels of antibiotic resistance were recorded from bacteria isolated from vended food and soil in Kangaru Market compared to Embu Town. The presence of antibiotic-resistant pathogens in street vended foods has been recently reported in Kisumu, Kenya [23]. An earlier study in Kibera, Kenya, reported that food and the environment are reservoirs of antibiotic resistance [24]. Food contaminated with antibiotic-resistant bacteria is a threat to public health as it encourages persistence and dissemination of resistance determinants [25]. The higher level of antibiotic resistance recorded in the Kangaru Market may be due to laxity in the enforcement of public health measures. Resistance to gentamycin and streptomycin which are administered by intramuscular injection was $48.57 \%$ and $32.86 \%$, respectively. The intramuscular mode of administration possibly limits the use of these antibiotics and thus contributes to the preservation of the antimicrobial activity [26]. In the present study, the frequency of antibiotic resistance to oral antibiotics was high and ranged from $72.86 \%$ for amoxicillin to $81.43 \%$ for nalidixic acid. The high level of resistance to oral antibiotics is possibly due to availability, convenient mode of administration, broad-spectrum activity, and affordability.

Fish samples in the present study had the highest $(18.52 \%)$ proportion of antibiotic-resistant isolates. The contamination of fish with antibiotic-resistant bacteria has been previously attributed to fecal contamination of water due to the unhygienic disposal of human waste [27]. Earlier studies reported contamination of water with bacteria that 
TABLE 4: Antibiosis effect of antibiotic-resistant bacteria isolated from vended food and soil on laboratory strains of bacteria.

\begin{tabular}{|c|c|c|c|c|c|c|}
\hline $\begin{array}{l}\text { Isolate } \\
\text { Number }\end{array}$ & Probable identity & Location & Sample & E. coli ATCC 35218 & S. aureus ATCC 1026 & K. pneumoniae ATCC 35218 \\
\hline KC430 & Pseudomonas spp. & Kangaru & Chips & $0.00 \pm 0.00^{\mathrm{b}}$ & $0.00 \pm 0.00^{\mathrm{b}}$ & $14.33 \pm 0.33^{\mathrm{a}}$ \\
\hline KC67 & $P$. aeruginosa & Kangaru & Chips & $0.00 \pm 0.00^{\mathrm{b}}$ & $0.00 \pm 0.00^{\mathrm{b}}$ & $10.00 \pm 0.00^{\mathrm{b}}$ \\
\hline KF395 & Yersinia spp. & Kangaru & Fish & $0.00 \pm 0.00^{\mathrm{b}}$ & $0.00 \pm 0.00^{\mathrm{b}}$ & $9.67 \pm 0.33^{b}$ \\
\hline KFR217 & Klebsiella spp. & Kangaru & Fruit & $0.00 \pm 0.00^{\mathrm{b}}$ & $0.00 \pm 0.00^{\mathrm{b}}$ & $7.00 \pm 0.00^{c}$ \\
\hline KFR38 & B. pacificus & Kangaru & Fruit & $0.00 \pm 0.00^{\mathrm{b}}$ & $8.33 \pm 2.33^{\mathrm{b}}$ & $13.67 \pm 0.33^{\mathrm{a}}$ \\
\hline KMP337 & C. freundii & Kangaru & Meat pie & $0.00 \pm 0.00^{\mathrm{b}}$ & $16.33 \pm 0.88^{\mathrm{a}}$ & $0.00 \pm 0.00^{\mathrm{d}}$ \\
\hline KS260 & E. coli & Kangaru & Soil & $7.00 \pm 0.00^{\mathrm{a}}$ & $0.00 \pm 0.00^{\mathrm{b}}$ & $9.00 \pm 0.00 b^{c}$ \\
\hline KSM63 & S. sonnei & Kangaru & Samosa & $0.00 \pm 0.00^{\mathrm{b}}$ & $0.00 \pm 0.00^{\mathrm{b}}$ & $10.33 \pm 1.33^{\mathrm{b}}$ \\
\hline KVS271 & Pseudomonas spp. & Kangaru & Vegetable salad & $0.00 \pm 0.00^{\mathrm{b}}$ & $0.00 \pm 0.00^{\mathrm{b}}$ & $7.00 \pm 0.00^{c}$ \\
\hline TS380 & Salmonella spp. & Town & Soil & $0.00 \pm 0.00^{\mathrm{b}}$ & $0.00 \pm 0.00^{\mathrm{b}}$ & $11.00 \pm 0.58^{\mathrm{b}}$ \\
\hline TS572 & B. subtilis & Town & Soil & $0.00 \pm 0.00^{\mathrm{b}}$ & $0.00 \pm 0.00^{\mathrm{b}}$ & $14.00 \pm 0.58^{\mathrm{a}}$ \\
\hline$P$ value & & & & 0.001 & 0.001 & 0.001 \\
\hline $\mathrm{CV}$ & & & & 0.00 & 76.76 & 9.03 \\
\hline LSD & & & & 0.00 & 3.80 & 2.54 \\
\hline
\end{tabular}

Mean with SE for antibiosis activity of the isolates, where the means with the same letters are not significantly different as indicated by Tukey's HSD test $(P<0.05)$.

Table 5: Risk groups of antibiotic-resistant bacteria isolated from vended foods and soil in Embu Town and Kangaru Market.

\begin{tabular}{|c|c|c|c|c|c|c|c|}
\hline $\begin{array}{l}\text { Isolate } \\
\text { no. }\end{array}$ & Location & Sample & Identity & $\begin{array}{c}\% \\
\text { similarity }\end{array}$ & $\begin{array}{c}\text { Resistant to } \\
\text { antibiotics }(n)\end{array}$ & $\begin{array}{c}\text { Resistance genes detected } \\
\text { by PCR }\end{array}$ & Riskgroup \\
\hline KFR42 & Kangaru & Fruit & B. amyloliquefaciens & 99.44 & 6 & - & 1 \\
\hline KFR38 & Kangaru & Fruit & B. pacificus & 99.1 & 6 & StrB & 1 \\
\hline KS65 & Kangaru & Soil & P. benzoelyticum & 99.57 & 3 & - & 1 \\
\hline KC75 & Kangaru & Chips & B. wiedmannii & 99.51 & 3 & - & 2 \\
\hline KF101 & Kangaru & Fish & B. velezensis & 97.86 & 4 & - & 1 \\
\hline KVS120 & Kangaru & $\begin{array}{l}\text { Vegetable } \\
\text { salad }\end{array}$ & B. subtilis & 97.94 & 8 & - & 1 \\
\hline KFR222 & Kangaru & Fruit & B. mobilis & 99.83 & 5 & ${ }^{B l a}$ TEM & 2 \\
\hline KF463 & Kangaru & Fish & B. anthracis & 99.43 & 7 & StrB, ${ }^{B l a}$ TEM & 1 \\
\hline TFR329 & Town & Fruit & B. proteolyticus & 97.18 & 7 & Str B, Dfr A & 1 \\
\hline KS109 & Kangaru & Soil & L. parviboronicapiens & 90.83 & 7 & $A m p$ & 1 \\
\hline KS104 & Kangaru & Soil & V. phasianinus & 99.31 & 3 & - & 1 \\
\hline KMP95 & Kangaru & $\begin{array}{l}\text { African } \\
\text { sausage }\end{array}$ & S. marcescens & 95.39 & 6 & Dfr A, FloR & 3 \\
\hline KS606 & Kangaru & Soil & B. anthracis & 100 & 5 & Tet A & 3 \\
\hline TS572 & Town & Soil & B. subtilis & 97.51 & 5 & Str B, ${ }^{\text {Bla }}$ TEM, FloR & 1 \\
\hline
\end{tabular}

- ,resistance gene not detected.

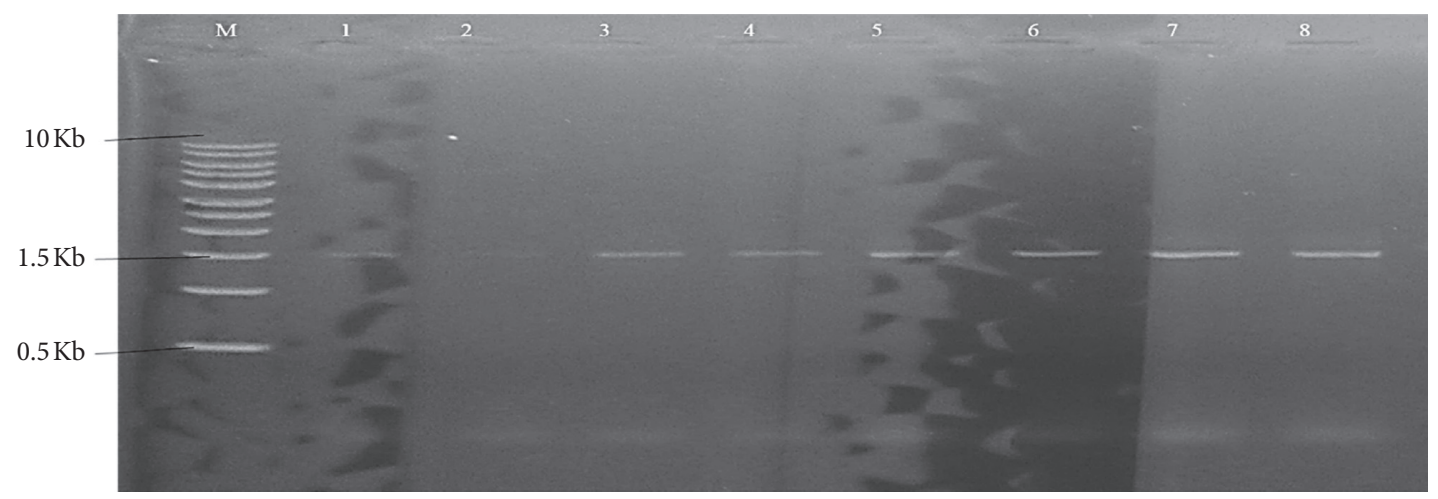

FIgURE 5: Amplification of bacterial 16S rRNA gene. $M$ is the molecular marker (1kb plus ladder); lane 1, isolate KVS68; 2, KMP45; 3, KC75; 4, TS380; 5, KVS214; 6, KFR38; 7, KS376; 8, KMP253. 


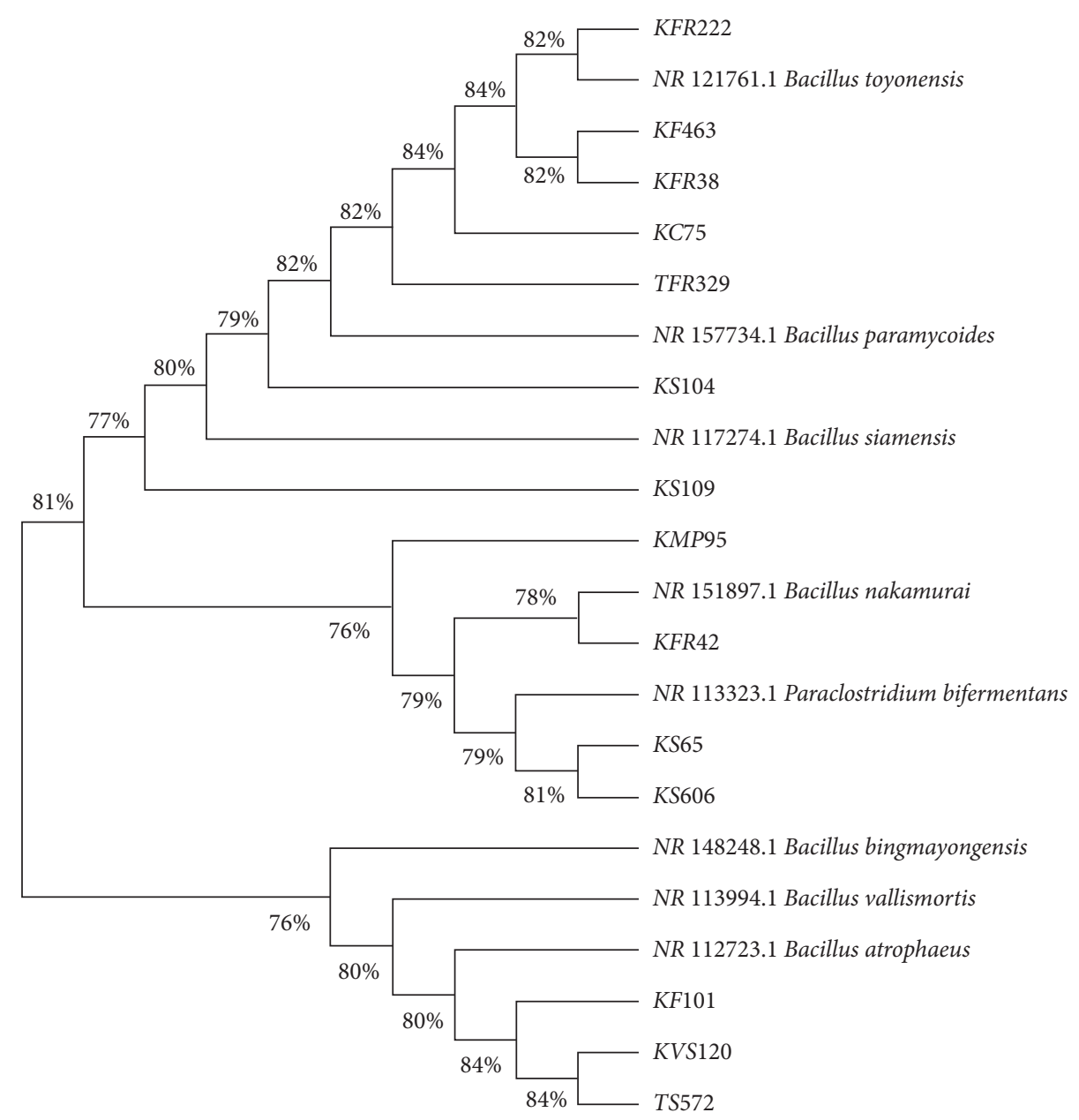

Figure 6: Phylogenetic relationships of bacterial isolates from vended food and soil in Embu Town and Kangaru Market. The evolutionary history was inferred through the neighbor-joining method. Isolates from the present study are presented in the phylogenetic tree using the isolate number codes.

were multidrug resistant to sulphamethoxazole/trimethoprim, ampicillin, and tetracycline [24, 28]. The present study confirmed that treated water from EWASCO that is used by most of the people in Embu Town and Kangaru Market was free from contamination by bacteria at the time of sampling. Therefore, it is unlikely that antibiotic resistance in this study originated from EWASCO water.

The current study isolated antibiotic-resistant bacteria from vegetable salad and African sausages, which are mostly homemade. Contamination of smokies, which are also a kind of sausage that is factory made and packed, with antibiotic-resistant bacteria was low. Postharvest contamination of vegetables and cross-contamination of African sausages are a major risk factor for disseminating antibiotic resistance [29, 30]. Soil samples from both Embu Town and Kangaru Market recorded a higher proportion of antibiotic-resistant isolates compared to food samples. In a previous study, anthropogenic activities such as long-term manure application were shown to increase antibiotic-resistant bacteria in soil [31]. Such activities were not deduced in the present study. Isolation of Pseudomonas spp. from both soil and fish in Kangaru
Market indicates the possibility of food contamination originating from the environment.

The present study showed that antibiotic resistance was encoded by TetA, ${ }^{B l a}$ TEM, StrB, DfrA, Amp, and FloR genes. In this study, genes encoding streptomycin resistance $S \operatorname{tr} B$ were detected in only nine out of the 23 isolates that were resistant to streptomycin. The $\operatorname{Str} B$ genes are known to be encoded by both transposons and plasmids [32]. The present study did not examine transposon-encoded antibiotic resistance, which could explain the detection of StrB in fewer isolates. Five of the nine isolates positive for FloR genes were isolated from food. This could be due to the use of chloramphenicol for domestic purposes [33]. Contamination of food by antibiotic-resistant bacteria could become a major threat to public health since the antibiotic resistance determinants can be transferred to pathogenic bacteria thus complicating the treatment of bacterial infections.

Sequencing of the $16 \mathrm{~S}$ rRNA gene revealed that most of the antibiotic-resistant bacterial food contaminants detected in this study belonged to the genus Bacillus. Although previous studies have associated Bacillus with food spoilage [34], isolation of $B$. wiedmannii, a risk 
group 2 from food, and B. anthracis, a risk group 3 organism from the soil, is of public health concern. The pathogen $B$. anthracis can cause the death of both human beings and animals [35] (https://my.absa.org/tiki-index). Since $S$. marcescens occurs naturally in soil and water, it can cause urinary, respiratory, meningitis, pneumonia, septicemia, and endocarditis infections in man. Isolation of $S$. marcescens from the African sausage in this study could be a result of contamination during preparation, as African sausages are mostly homemade. The presence of S. marcescens a risk group 3 organism [7] in food is of grave concern.

Although isolate TS572 affiliated to B. subtilis was resistant to five antibiotics, it also had antimicrobial activity against K. pneumoniae ATCC 49619. This is not surprising as B. subtilis from soil have been reported to produce antimicrobial agents [36]. KMP337 affiliated to $C$. freundii recorded the highest antibiosis activity against $S$. aureus ATCC 1026. Previous studies associated $C$. freundii with multiple antibiotic resistance but not the production of antimicrobial agents [37]. The present study isolated B. amyloliquefaciens from fruits in Kangaru Market. In a previous study, B. amyloliquefaciens was isolated from banana fruit surfaces and was shown to produce antimicrobial activity against crown root causing pathogens. Thus, B. amyloliquefaciens could be explored further for antimicrobial agents [38]. Isolation of bacteria with antimicrobial activity in the present study supports the need for innovative screening strategies in the search for new antimicrobial agents. Most of the bacteria isolated in the current study were able to produce extracellular enzymes. Other studies have suggested that isolates that exhibit high enzymatic activity also possess high antibiosis activity; thus, these processes could be occurring concurrently $[18,39]$.

\section{Conclusions}

This study showed that vended food and the soil in Embu Town and Kangaru Market contain bacterial food contaminants that are resistant to commonly used antibiotics. The study further confirmed that the bacteria harbored antibiotic resistance determinants TetA, ${ }^{B l a}$ TEM, StrB, DfrA, $A m p$, and FloR. Some of the bacterial food contaminants isolated in this study are risk group 2 and 3 organisms which is a public health concern. More resistance was recorded in the bacteria originating from Kangaru Market as compared to Embu Town. Overall, vended food in Kangaru Market may pose a health risk to the public if mitigating measures are not put in place.

\section{Data Availability}

Additional data for this manuscript have been provided as supplementary material.

\section{Disclosure}

A license to conduct the research was obtained from the National Commission for Science, Technology and Innovation (NACOSTI) in Kenya. Authorization to collect samples was granted by the Public Health Ministry of Embu County.

\section{Conflicts of Interest}

The authors declare that there are no conflicts of interest in the publication of this paper.

\section{Acknowledgments}

This research was supported by the University of Embu.

\section{Supplementary Materials}

Supplementary Table 1: morphological and biochemical characterization of antibiotic-resistant bacteria isolated from Embu Town and Kangaru Market in 2018. Supplementary Table 2: drug sensitivity interpretation of zone of inhibition diameter based on CLSI (Standard and Testing Guidelines 2018). Supplementary Table 3: enzymatic activity of the bacteria isolated from vended food and soil in Embu Town and Kangaru Market. Supplementary Figure 1: enzymatic activity of the antibiotic-resistant bacterial isolates isolated from fast food and the soil in Embu Town and Kangaru Market. (Supplementary Materials)

\section{References}

[1] I. C. Oladipo and O. D. Adejumobi, "Incidence of antibiotic resistance in some bacterial pathogens from street vended food in Ogbomoso, Nigeria," Pakistan Journal of Nutrition, vol. 9, no. 11, pp. 1061-1068, 2010.

[2] M. Jahan, M. Rahman, M. Rahman et al., "Microbiological safety of street-vended foods in Bangladesh," Journal of Consumer Protection and Food Safety, vol. 13, no. 3, pp. 257-269, 2018.

[3] S. Omulo, S. M. Thumbi, M. K. Njenga, and D. R. Call, “A review of 40 years of enteric antimicrobial resistance research in Eastern Africa: what can be done better?" Antimicrobial Resistance \& Infection Control, vol. 4, no. 1, p. 1, 2015.

[4] C. T. Walsh and M. A. Fischbach, "New ways to squash superbugs," Scientific American, vol. 301, no. 1, pp. 44-51, 2009.

[5] I. A. N. Okeke, S. Kariuki, G. J. Dinant, C. Driessen, and E. E. Stobberingh, "Antibiotic resistance of faecal Escherichia coli from healthy volunteers from eight developing countries," Journal of Antimicrobial Chemotherapy, vol. 54, no. 5, pp. 952-955, 2004.

[6] G. Githuka, "Notes from the field: ongoing cholera outbreak-Kenya, 2014-2016," Morbidity and Mortality Weekly Report, vol. 65, 2016.

[7] ABSA, "Risk group database," 2016.

[8] K. Njiru Peter, O. Omuterema Stanley, and D. B. Lilechi, "Sanitation in Relation to Prevalence of Waterborne Diseases in Mbeere," American Society for Microbiology (ASM), Embu County, Kenya, 2016.

[9] W. C. Hazeleger and R. R. Beumer, "Microbial quality of raw horse milk," International Dairy Journal, vol. 63, pp. 59-61, 2016.

[10] J. G. Cappuccino and N. Sherman, "Techniques for isolation of pure cultures," Cultural and morphological characteristics of microorganisms, vol. 6, pp. 13-23, 2002. 
[11] N. A. Ledeboer and G. V. Doern, Haemophilus Manual of Clinical Microbiologypp. 667-684, American Society for Microbiology (ASM), New York, NY, USA, 11th edition, 2015.

[12] L. S. Clesceri, A. E. Greenberg, A. D. Eaton, E. W. Rice, and M. A. H. Franson, "Standard methods for the examination of water and wastewater," Journal of Advanced Biotechnology and Bioengineering, vol. 628, 2005.

[13] T. Sharma, A. Sharma, and S. S. Kanwar, "Purification and characterization of an extracellular high molecular mass esterase from Bacillus pumilus," Journal of Advanced Biotechnology and Bioengineering, vol. 4, no. 1, pp. 9-16, 2016.

[14] C. Torres, D. O. Leandro, P. Jamile, and Q. Pereira, "Screening of bacteria for protease production and feather degradation," Waste and Biomass Valorization, vol. 4, 2016.

[15] G. L. Furtado and A. A. Medeiros, "Single-disk diffusion testing (kirby-bauer) of susceptibility of Proteus mirabilis to chloramphenicol: significance of the intermediate category," Waste and Biomass Valorization, vol. 198, 2015.

[16] P. Standards and A. S. Testing, "M100 performance standards for antimicrobial," 2018.

[17] K. J. M. Nuneza, D. M. M. Abella, L. L. Maratas, and O. M. Nuneza, "Antibiotic phenotypic profile of fecal Escherichia coli from pediatric patients of adventist medical center, Iligan city, Philippines," Advances in Environmental Biology, vol. 9, pp. 129-138, 2015.

[18] J. O. Neondo, A. E. Alakonya, and R. W. Kasili, "Screening for potential Striga hermonthica fungal and bacterial biocontrol agents from suppressive soils in Western Kenya," BioControl, vol. 62, no. 5, pp. 705-717, 2017.

[19] J. Sambrook and D. W. Russell, "Molecular clonning: a laboratory manual," Molecular Clonning: A Laboratory Manual, vol. 62, 2001.

[20] H. C. Bimboim and J. Doly, "A rapid alkaline extraction procedure for screening recombinant plasmid DNA," Nucleic Acids Research, vol. 7, no. 6, pp. 1513-1523, 1979.

[21] J. Sambrook, E. F. Fritsch, T. Maniatis, and T. SambrookFritsch-Maniatis, "Molecular cloning: a laboratory manual; (prepared for use in the CSH courses on the molecular cloning of eukaryotic genes)," Cold Spring Harbor Laboratory Press, vol. 7, 1989.

[22] J. Sambrook and D. W. Russell, "Molecular Cloning: A Laboratory Manual," Cold Spring Harbor Laboratory Press, New York, NY, USA, 3rd edition, 2001.

[23] D. M. Onyango, "Source tracking and antibiotic resistance patterns of selected pathogenic bacteria isolated from street vended food in Kisumu county, Kenya," EC Pharmacology and Toxicology, vol. 7, pp. 585-596, 2019.

[24] N. C. Muhonja, N. Budambula, J. Kiiru, and S. Kariuki, "Characterization of antibiotic resistance in environmental enteric pathogens from Kibera slum," EC Pharmacology and Toxicology, vol. 4, pp. 46-54, 2012.

[25] F. Disease, B. Epidemiology, and R. Group, "Who estimates of the global burden of foodborne diseases," 2015.

[26] E. Muñoz-atienza, "Antimicrobial activity, antibiotic susceptibility and virulence factors of lactic acid bacteria of aquatic origin intended for use as probiotics in aquaculture," 2013.

[27] A. W. Sifuna and D. M. Onyango, "Source attribution of Salmonella and Escherichia coli contaminating lake victoria fish in Kenya," African Journal of Tropical Hydrobiology and Fisheries, vol. 16, no. 1, pp. 39-47, 2018.

[28] J. K. Too, W. Kipkemboi Sang, Z. Ng'ang'a, and M. O. Ngayo, "Fecal contamination of drinking water in Kericho District,
Western Kenya: role of source and household water handling and hygiene practices," Journal of Water and Health, vol. 14, no. 4, pp. 662-671, 2016.

[29] E. K. Kutto, M. W Ngigi, N Karanja et al., "Bacterial contamination of Kale (Brassica oleracea acephala) along the supply chain in Nairobi and its environment," East African Medical Journal, vol. 88, no. 2, pp. 46-53, 2011.

[30] W. H. Karoki, D. N. Karanja, L. C. Bebora, and L. W. Njagi, "Isolation, characterization, and quantification of bacteria from African sausages sold in Nairobi County, Kenya," International Journal of Food Sciences and Nutrition, vol. 2018, 2018.

[31] L. Han, L. Cai, H. Zhang, Z. Long, Y. Yu, and H. Fang, "Development of antibiotic resistance genes in soils with ten successive treatments of chlortetracycline and ciprofloxacin," Environmental Pollution, vol. 253, pp. 152-160, 2019.

[32] J. L. Vanneste, M. D. Voyle, J. Yu, D. A. Cornish, R. J. Boyd, and G. F. Mclaren, "Copper and streptomycin resistance in Pseudomonas strains isolated from pipfruit and stone fruit orchards in New Zealand," 2008.

[33] J. Lu, "Spread of the florfenicol resistance floR gene among clinical Klebsiella pneumoniae isolates in China, Antimicrob," Antimicrobial Resistance \& Infection Control, vol. 7, no. 1, p. 127, 2018.

[34] J. Owusu-Kwarteng, A. Wuni, F. Akabanda, K. Tano-Debrah, and L. Jespersen, "Prevalence, virulence factor genes and antibiotic resistance of Bacillus cereus sensu lato isolated from dairy farms and traditional dairy products," BMC Microbiology, vol. 17, no. 1, p. 65, 2017.

[35] M. C. Erickson, "Internalization of fresh produce by foodborne pathogens," Annual Review of Food Science and Technology, vol. 3, no. 1, pp. 283-310, 2012.

[36] M. J. Torres, G. Petroselli, M. Daz, R. Erra-Balsells, and M. C. Audisio, "Bacillus subtilis subsp. subtilis CBMDC3f with antimicrobial activity against Gram-positive foodborne pathogenic bacteria: UV-MALDI-TOF MS analysis of its bioactive compounds," World Journal of Microbiology and Biotechnology, vol. 31, no. 6, pp. 929-940, 2015.

[37] C. Pepperell, J. V. Kus, M. A. Gardam, A. Humar, and L. L. Burrows, "Low-virulence Citrobacter species encode resistance to multiple antimicrobials," Antimicrobial Agents and Chemotherapy, vol. 46, no. 11, pp. 3555-3560, 2002.

[38] D. G. Alvindia and K. T. Natsuaki, "Biocontrol activities of Bacillus amyloliquefaciens DGA14 isolated from banana fruit surface against banana crown rot-causing pathogens," Crop Protection, vol. 28, no. 3, pp. 236-242, 2009.

[39] O.-A. Sicuia, I. Grosu, F. Constantinescu, C. Voaides, and C. P. Cornea, "Enzymatic and genetic variability in Bacillus spp. strains with plant beneficial qualities," AgroLife Scientific Journal, vol. 4, pp. 124-131, 2015. 